\title{
COVERINGS OF CUBIC GRAPHS AND 3-EDGE COLORABILITY
}

\author{
LEONID PlaChTA \\ AGH University of Science and Technology \\ Kraków, Poland \\ e-mail: dept25@gmail.com
}

\begin{abstract}
Let $h: \tilde{G} \rightarrow G$ be a finite covering of 2-connected cubic (multi)graphs where $G$ is 3-edge uncolorable. In this paper, we describe conditions under which $\tilde{G}$ is 3 -edge uncolorable. As particular cases, we have constructed regular and irregular 5-fold coverings $f: \tilde{G} \rightarrow G$ of uncolorable cyclically 4-edge connected cubic graphs and an irregular 5-fold covering $g: \tilde{H} \rightarrow H$ of uncolorable cyclically 6 -edge connected cubic graphs.

In [13], Steffen introduced the resistance of a subcubic graph, a characteristic that measures how far is this graph from being 3-edge colorable. In this paper, we also study the relation between the resistance of the base cubic graph and the covering cubic graph.
\end{abstract}

Keywords: uncolorable cubic graph, covering of graphs, voltage permutation graph, resistance, nowhere-zero 4-flow.

2010 Mathematics Subject Classification: 05C15, 05C10.

\section{REFERENCES}

[1] R. Diestel, Graph Theory (Springer, Berlin, Heidelberg, 2010). doi:10.1007/978-3-642-14279-6

[2] M.A. Fiol, G. Mazzuoccolo and E.Steffen, On measures of edge-uncolorability of cubic graphs: A brief survey and some new results, 23 Feb 2017. arXiv:1702.07156v1[math.CO]

[3] M. Ghebleh, The circular chromatic index of Goldberg snarks, Discrete Math. 307 (2007) 3220-3225. doi:10.1016/j.disc.2007.03.047

[4] J.L. Gross and T.W. Tucker, Generating all graph coverings by permutation voltage assignments, Discrete Math. 18 (1977) 273-283.

doi:10.1016/0012-365X(77)90131-5 
[5] J.L. Gross and T.W. Tucker, Topological Graph Theory (Dover Publications Inc., New York, 2012).

[6] J. Hägglund, On snarks that are far from being 3-edge colorable, Electron. J. Combin. 23 (2016) \#P2.6.

[7] M. Kochol, Snarks without small cycles, J. Combin. Theory Ser. B 67 (1996) 34-47. doi:10.1006/jctb.1996.0032

[8] R. Lukot'ka, E. Máčajová, J. Mazák and M. Škoviera, Small snarks with large oddness, Electron. J. Combin. 22 (2015) \#P1.51.

[9] E. Máčajová and M. Škoviera, Irreducible snarks of given order and cyclic connectivity, Discrete Math. 306 (2006) 779-791. doi:10.1016/j.disc.2006.02.003

[10] W. McCuaig, Edge reductions in cyclically $k$-connected cubic graphs, J. Combin. Theory Ser. B 56 (1992) 16-44. doi:10.1016/0095-8956(92)90004-H

[11] B. Mohar, E. Steffen and A. Vodopivec, Relating embeddings and coloring properties of snarks, Ars Math. Contemp. 1 (2008) 169-184. doi:10.26493/1855-3974.49.b88

[12] R. Nedela and M. Škoviera, Decompositions and reductions of snarks, J. Graph Theory 22 (1996) 253-279. doi:10.1002/(SICI)1097-0118(199607)22:3〈253::AID-JGT6〉3.0.CO;2-L

[13] E. Steffen, Measurements of edge-uncolorability, Discrete Math. 280 (2004) 191214. doi:10.1016/j.disc.2003.05.005

[14] V.G. Vizing, Critical graphs with given chromatic class, Diskret. Analiz. 5 (1965) 9-17 (in Russian).

Received 18 October 2017 Revised 10 September 2018 Accepted 29 October 2018 